\title{
Particle-stabilised foams: structure and aging
}

\author{
Antonio Stocco, $\dagger^{* a}$ Francisco Garcia-Moreno, ${ }^{b c}$ Ingo Manke, ${ }^{b}$ John Banhart ${ }^{b c}$ and Dominique Langevin ${ }^{a}$ \\ Received 26th March 2010, Accepted 22nd September 2010 \\ DOI: 10.1039/c0sm00166j
}

We show that aqueous foams stabilised by nanoparticles can be easily imaged using an X-ray

laboratory source. We have used hydrophobically modified silica nanoparticles that confer to the foam

a remarkable stability. The X-ray tomography observations were compared with the information

obtained using a multiple light scattering technique. Both techniques confirm that provided the

concentration of particles in bulk water is high enough, the bubble size evolves little with time. X-Ray

tomography revealed the presence of two populations of bubbles, small bubbles which size tends to

decrease with time and large bubbles which number tends to increase with time. This behaviour could

arise from an arrested coarsening process. The results demonstrate the great potential of the two

techniques and of their combination for foam studies.

\section{Introduction}

Aqueous foams are presently the object of much interest, both due to the progresses in theoretical modelling and to the numerous potential applications, in particular as precursors for new materials. ${ }^{1}$ It is difficult to characterise bubble size and liquid volume fraction in foams, as they are frequently opaque. Most of the characterisations have been made on quasi-two dimensional foams, e.g. foams made of a single layer of bubbles. Studies of truly three-dimensional foams are less numerous, the most widely used technique to date being diffusing wave spectroscopy (DWS), based on the properties of the light multiply scattered by the foam. ${ }^{2-5}$ Although this method can include the study of the spectrum of the scattered light, the measurement of the diffusely transmitted (or back-scattered) intensity itself contains interesting information about bubble size and liquid fraction. A second technique mostly used to date with metallic foams is X-ray tomography. ${ }^{6}$ Contrary to light, foams are transparent to X-rays, making possible imaging inside the foam. This technique has recently been applied to surfactant foams, using a synchrotron source., ${ }^{7,8}$

Because nanoparticles offer a better contrast to X-ray than surfactants, we decided to apply the technique to aqueous foams stabilised by particles using a laboratory source. We have acquired in the recent years expertise on aqueous foams stabilised by silica nanoparticles. ${ }^{9,10}$ These particles are hydrophobically modified, in order to ensure large enough adsorption energies at the air-water surface. The X-ray tomography observations were compared with the information obtained using a multiple light scattering technique. In this paper we describe the experiments and discuss the results, which open interesting perspectives for further studies.

${ }^{a}$ Laboratoire de Physique des Solides, Université Paris-Sud, UMR CNRS 8502, Bâtiment 510,91405 Orsay cedex, France.E-mail: antonio.stocco@, mpikg.mpg.de

${ }^{b}$ Helmholtz-Zentrum Berlin für Materialien und Energie, Hahn-MeitnerPlatz 1, 14109 Berlin, Germany

'Technische Universität Berlin, Hardenbergstrasse 36, 10623 Berlin, Germany

$\dagger$ Present address: Max Planck Institute of Colloids and Interfaces, 14476 Golm, Potsdam, Germany.

\section{Material, preparation and methods}

\subsection{Material}

Silica nanoparticles were provided by Wacker-Chemie (Germany) and were used as received. The hydrophobicity of the particles was controlled by reacting the surface silanol groups of silica with dichlorodimethylsilane by the manufacturer. In this work, we used particles such that $34 \%$ of the surface $\mathrm{SiOH}$ groups remained untreated. The primary particles are spheres of approximately $20 \mathrm{~nm}$ diameter and are irreversibly aggregated into clusters of approximately $170 \mathrm{~nm}$ diameter, as measured in a previous light scattering study. ${ }^{10}$ The dispersions were prepared by adding step-by-step the amorphous fumed silica powder into water up to a final bulk concentration of $c=1 \mathrm{wt} \%$, while using a low amount $(2 \mathrm{wt} \%)$ of ethanol in the first stages to facilitate particle wetting by water. The dispersion was sonicated for one hour using an ultrasonic probe (Ultrasonic Processor) operating at $20 \mathrm{kHz}$ with $70 \%$ of the maximum amplitude in order to obtain a stable dispersion and to break the large particle aggregates. The dispersion was resonicated and treated again for 5 minutes just before each foaming experiment.

Water used in the experiments was ultra-purified water from a Millipore-Q instrument (resistivity $=18 \mathrm{M} \Omega \mathrm{cm}$ ).

\subsection{Foam production}

Foam samples were prepared either by turbulent mixing or shaking. The liquid volume fraction $\varepsilon$ was evaluated by image analysis (monitoring the position of the separation between foam and drained liquid).

For turbulent mixing we used an apparatus (described elsewhere ${ }^{11}$ ) that allows obtaining a large amount of relatively dry foam in a very short time. Typical initial liquid fractions $\varepsilon_{0}$ for turbulently generated foams range from 0.16 to $0.20 .{ }^{11} \mathrm{We}$ could generate foams with a larger liquid fraction $\left(\varepsilon_{0} \approx 0.34\right)$ only from dispersions with $c=0.1 \mathrm{wt} \%$. The final liquid fraction observed with this preparation method is $\varepsilon_{\mathrm{f}} \approx 0.09$ for all concentrations studied. Foam cells used for multiple light scattering experiments were filled directly from the outlet tube of the turbulent mixing apparatus. For the X-ray tomography experiment, the foam was 
Table 1 Aqueous volume fraction in the foams studied

\begin{tabular}{llll}
\hline Method & $c / \mathrm{wt} \%$ & $\varepsilon_{0}$ & $\varepsilon_{\mathrm{f}}$ \\
\hline Turbulent mixing & $0.1-0.7$ & $0.16-0.20^{a}$ & 0.09 \\
Shaking & $0.3-0.9$ & 0.34 & 0.21 \\
${ }^{a}$ For $c=0.1 \mathrm{wt} \%, \varepsilon_{0}=0.34$. & & \\
\hline
\end{tabular}

stored in a $50 \mathrm{~mL}$ syringe before being transferred into a container made of a $50 \mu \mathrm{m}$ thick capton cylinder $(10 \mathrm{~mm}$ diameter and $40 \mathrm{~mm}$ height) closed at the ends by plastic caps.

Foams obtained by vigorous shaking by hand for 1-2 minutes were relatively wet, with an average initial liquid fraction of $\varepsilon_{0} \approx 0.34$ and a final liquid fraction after $10^{6} \mathrm{~s}$ of $\varepsilon_{\mathrm{f}} \approx 0.21$. Using this production method for dispersions with $c=0.1 \mathrm{wt} \%$, we could not generate an amount of foam sufficient for further studies. For the light scattering experiments, the foams were placed into cylindrical plastic containers $(27 \mathrm{~mm}$ interior diameter and $120 \mathrm{~mm}$ height). For the X-ray tomography experiments, the foams were first placed into a $250 \mathrm{~mL}$ container and were left there for 10 minutes to allow the foam to drain, after which they were transferred into the capton cylinder container.

Hereafter, we will refer to foams prepared by turbulent mixing as "dry" and the ones prepared by shaking as "wet". The foam properties are summarized in Table 1 .

\subsection{X-Ray tomography}

In order to analyze the foam structure and morphology, X-ray tomography was performed using an X-ray lab ( $\mu-\mathrm{CT})$ setup. The equipment is based mainly on an X-ray source, a sample rotation stage and a flat panel detector. We used a micro-focus source from Hamamatsu (L8121-03) that has a W target. X-Ray energies up to $150 \mathrm{keV}$ are available with a variable spot size of $5-50 \mu \mathrm{m}$. For our measurements, an energy of $80 \mathrm{keV}$, a spot size of $7 \mu \mathrm{m}$ and a current of $100 \mu \mathrm{A}$ were chosen. Imaging of the sample is based on absorption contrast between the fluid and the gas. The images are recorded by a $120 \times 120 \mathrm{~mm}^{2}$ $\left(50 \times 50 \mu \mathrm{m}^{2}\right.$ pixel size $)$ flat panel detector, also from Hamamatsu (C7942SK-05, GOS scintillator screen). The geometrical magnification of the cone beam tomography setup is given by the ratio between the source-to-detector distance $(300 \mathrm{~mm})$ and the source-to-sample distance $(60 \mathrm{~mm})$, in our case a factor of 5 . The spatial resolution in this configuration is therefore limited by the detector, with a pixel size corresponding to $10 \mu \mathrm{m}$ and a physical resolution of about $20 \mu \mathrm{m}$. A rotation table from Huber (one circle goniometer 408) is synchronized with the recording software, providing a stack of images when rotating the sample by $360^{\circ}$. The acquired images are later reconstructed into $3 \mathrm{D}$ volume datasets using a cone beam reconstruction algorithm built into the software Octopus. ${ }^{12}$ The $3 \mathrm{D}$ images are visualized with VGStudiomax. ${ }^{13}$ The quantitative analysis of the bubble size distribution is performed with Avizo. ${ }^{14}$ This includes several 3D image treatment steps such as filtering, binarisation, segmentation, etc. More details on the application of tomography to foams can be found in ref. 6. The bubble diameter distribution is in general well fit by a lognormal function, from which a mean diameter can be directly determined.
To obtain a higher spatial resolution, some samples were studied in the synchrotron X-ray tomography facility at the BAMline (Bessy, HZB, Germany). A high resolution setup was used that provides spatial resolutions down to $0.6 \mu \mathrm{m}$ with a pixel size of about $0.3 \mu \mathrm{m}$ (optical setup Optique Peter, Optical and Mechanical engineering France). A PCO4000 CCD camera with $4008 \times 2672$ pixels and a $50 \mu \mathrm{m}$ thick CWO scintillator screen were used. The X-ray beam was monochromatized with a double multilayer monochromator (WSi) that provides an energy resolution of $\Delta E / E \approx 10^{-2}$. An X-ray energy of $15 \mathrm{kV}$ (with 2 filters (Al: $0.5 \mathrm{~mm}$ and Be: $0.6 \mathrm{~mm}$ )) was chosen for optimal transmission and contrast. The principles of image acquisition are similar as for the lab CT. With the help of phase contrast (sample-detector distance of $300 \mathrm{~mm}$ ) we could reach high contrast with a resolution of about $2.5 \mu \mathrm{m}$ (with a pixel size of $1.1 \mu \mathrm{m}$ ). A filtered back-projection algorithm (Software Octopus) was used for tomographic reconstructions. Compared to X-ray lab $\mu-\mathrm{CT}$, synchrotron tomography provides a higher spatial resolution but a smaller analysis volume (several $\mathrm{mm}^{3}$ ): less bubbles are imaged and as a result, the statistical analysis of the bubble size distribution is less accurate.

All the experiments described in the paper were performed at room temperature $\left(\sim 22^{\circ} \mathrm{C}\right)$.

\subsection{Multiple light scattering}

In order to study the foam evolution we measured the multiply scattered light intensity $I_{\mathrm{s}}$ during foam aging. ${ }^{5}$ In this study, we adopted a transmission scheme. The source of radiation was a laser (Coherent, Compass 315M-100) working at $\lambda=533 \mathrm{~nm}$. The foam cell was a plastic cylinder $120 \mathrm{~mm}$ high and with an internal diameter of $27 \mathrm{~mm}$. The diffuse transmitted light was collected by an optical fibre (Oz Optics) equipped with a focus lens designed to collect a spot size comparable to the laser speckle. A photomultiplier (Hamamatsu) and a correlator (Correlator.com, Flex2k-12 $\times 2$ ) were used together with the software Flex2k. A CCD camera (Eurosoft) was positioned in the horizontal scattering plane at $\sim 10^{\circ}$ from the transmitted beam, and its objective was focused on the vertical axis of the cylindrical container in order to check for the foam integrity during the measurements. The scattering volume was related to the path of light in the foam and proportional to the diameter of the laser beam $(\sim 500 \mu \mathrm{m})$ and to the sample thickness. It is chosen above the macroscopic interface between the drained liquid and the foam. In order to follow the foam time evolution (usually up to $10^{5} \mathrm{~s}$ ), we usually performed 7 measurements of $10 \mathrm{~s}$, followed by 7 measurements of $120 \mathrm{~s}$ and 14 measurements of $7200 \mathrm{~s}$ durations. To test our apparatus, we measured $I_{\mathrm{s}}$ for a foam with known properties (Gillette Normal, UK). ${ }^{5}$ The results are reported in the Appendix.

For homogeneous aqueous foams, the multiply scattered light intensity $I_{\mathrm{s}}$ is proportional to $l^{*} / L$, where $l^{*}$ is the mean free path of light and $L$ is the foam container internal width. $l^{*}$ is directly proportional to the average bubble diameter $D$ and inversely proportional to the square root of foam liquid fraction $\varepsilon: I_{\mathrm{s}} \approx$ $D / \sqrt{ } \varepsilon \varepsilon^{2,5,15}$ Thus, the information given by $I_{\mathrm{s}}$ accounts for both changes of the average bubble radius and liquid fraction. The theory is valid, provided that the medium is homogeneous meaning that the material can be described by a single $l^{*}$ and the 
ratio bubble diameter/container thickness does not exceed $0.2(D / L<0.2){ }^{4,5}$

\section{Experimental results}

\subsection{Foam aging}

We studied the time evolution of "dry" aqueous foams using silica nanoparticles aqueous dispersions of bulk concentration $c=0.1,0.3,0.5$, and $0.7 \mathrm{wt} \%$. In Fig. 1 , we show the ratio between the multiply scattered intensity $I_{\mathrm{s}}$ and its initial value $I_{\mathrm{s}}{ }^{0}$ for the four concentrations studied.

For $c=0.1 \mathrm{wt} \%, I_{\mathrm{s}}$ remains roughly constant for the first 100 seconds and increases by a factor of 10 between $10^{2}$ and $10^{4} \mathrm{~s}$. For $c=0.3$ and $0.5 \mathrm{wt} \%$, the intensity increases less rapidly and reaches a limiting value around $10^{4} \mathrm{~s}$ and $10^{5} \mathrm{~s}$ respectively. The evolution in the first stages of aging is shown in Fig. 2 for $c=$ $0.3 \mathrm{wt} \%$ and reveals a small increase of $I_{\mathrm{s}}(\sim 10 \%)$ for the first 70 seconds. For the three concentrations, when a limit value of the intensity $\left(I_{\mathrm{S}} / I_{\mathrm{s}}{ }^{0}=c a\right.$. 10$)$ is reached, the ratio $D / L>0.2$; the medium cannot be considered optically thick anymore and the multiple scattering theory is no longer valid. For $c=0.7 \mathrm{wt} \%$, even at a foam age of $7 \times 10^{5} \mathrm{~s}(\sim 8$ days $), I_{\mathrm{s}}$ does not change appreciably and tends to saturate around $3 I_{\mathrm{s}}{ }^{0}$.

The average liquid fraction $\varepsilon$ remains almost constant for the first $70 \mathrm{~s}$ and decreases afterwards due to drainage from $0.16-0.2$ to 0.09 . The initial small increase of $I_{\mathrm{s}}$ seen in Fig. 2 is therefore due to a small increase in average bubble size $(\sim 10 \%)$. After $70 \mathrm{~s}$, the decrease of $\varepsilon$ also contributes to $I_{\mathrm{s}}: 1 / \sqrt{ } \varepsilon$ which increases by a factor of 2 for $0.1 \mathrm{wt} \%$ and 1.5 for the other concentrations. We did not therefore attempt to check the scaling law for coarsening ( $D \approx t^{1 / 2}$ ), as in the case of Gillette foam (see Appendix). For the very stable "dry" foam made with $c=0.7 \mathrm{wt} \%$, the mean bubble diameter is expected to have increased only by a factor 2 at a foam age $\approx 10^{6} \mathrm{~s}$. This is consistent with earlier results in which a rotating cell was used to suppress drainage. ${ }^{9}$

For "wet" foams, the multiply scattered intensity does not change significantly in time as shown in Fig. 1. For all the concentrations studied, $I_{\mathrm{s}}$ remains approximately constant until a foam age of $10^{5} \mathrm{~s}$. During the first $70 \mathrm{~s}$, the liquid fraction decreases from about 0.34 to 0.27 and the intensity should have

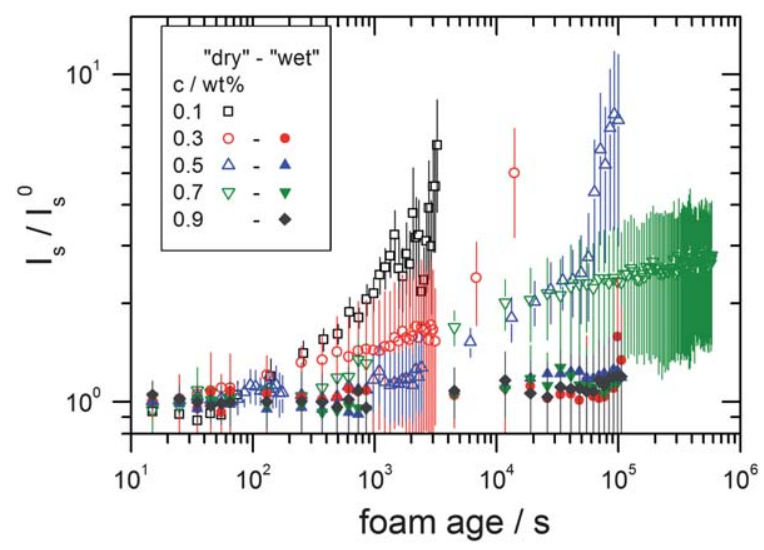

Fig. 1 Multiple scattered intensity ratio as a function of time for "dry" foams (open symbols) and "wet" foams (filled symbols) at different bulk concentrations (given in the legend).

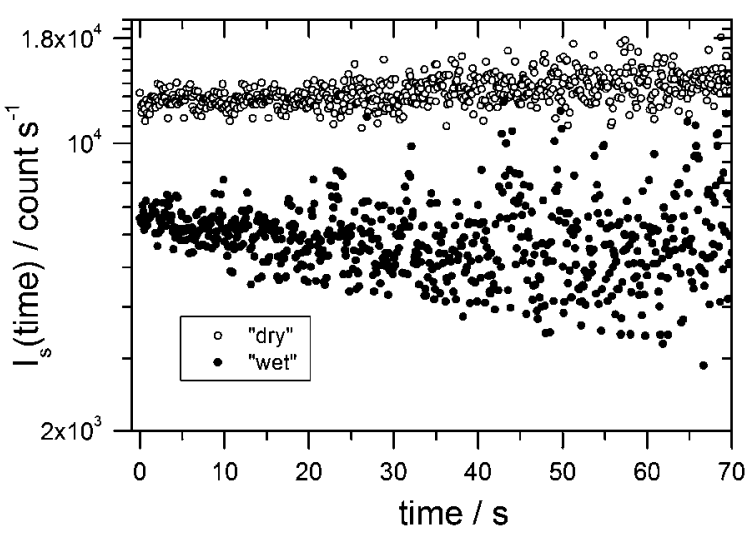

Fig. 2 Scattered intensity as a function of time for "dry" foams and "wet" foam (both at $c=0.3 \mathrm{wt} \%$ ) just after foam generation.

increased by about $10 \%$. This is unfortunately smaller than the error bars to be detected (Fig. 2). At long times, $\varepsilon$ decreases down to 0.21 , whereas the intensity does not change within error bars. Again the error bars are too large to detect such a small change.

\subsection{Foam structure}

The structure of the "wet" foams with $c=0.9 \mathrm{wt} \%$ for foam ages of $\sim 10^{4} \mathrm{~s}$ and $\sim 10^{5} \mathrm{~s}$ as obtained by $3 \mathrm{D}$ X-ray $\mu$-CT imaging is shown in Fig. 3. The foams are virtually sliced along the axis of the capton cylinder to facilitate the assessment of the foam structure. They exhibit a significant polydispersity, as expected for foams made by shaking. Comparison of Fig. 3(a) and (b) reveals that the foam structure after $\sim 10^{4} \mathrm{~s}$ with a mean bubble diameter of $150 \mu \mathrm{m}$ is more uniform than the structure after $\sim 10^{5} \mathrm{~s}$, where we find a smaller mean diameter of $40 \mu \mathrm{m}$ but also a significant population of bigger bubbles (Fig. 4). At this point it is necessary to remark that we are considering the arithmetic mean value in order to fit the distribution, and not the volume-weighted mean due to the lack of statistics.

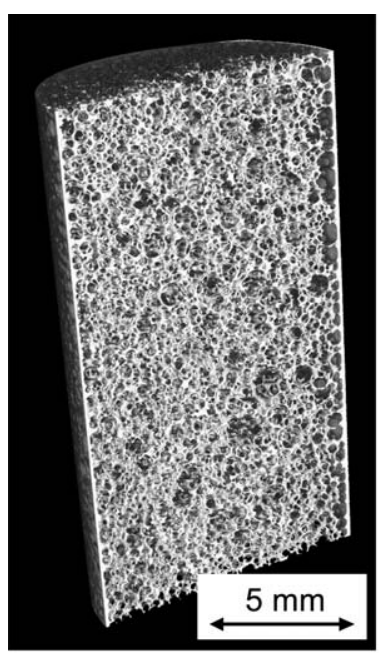

(a)

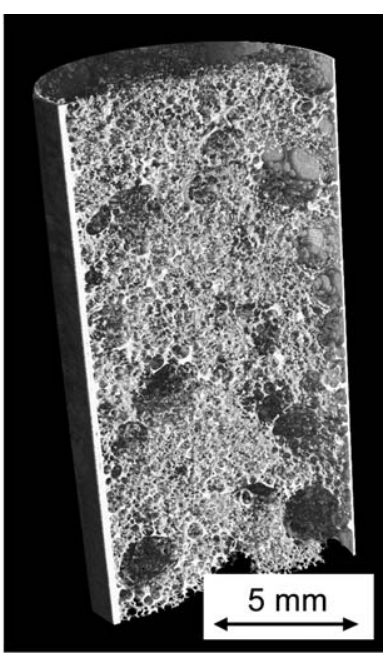

(b)
Fig. 3 3D X-ray $\mu$-CT image reconstructions of a "wet" foam obtained $(c=0.9 \mathrm{wt} \%)$ at foam ages $\approx 10^{4} \mathrm{~s}(\mathrm{a})$ and $\approx 10^{5} \mathrm{~s}(\mathrm{~b})$. 


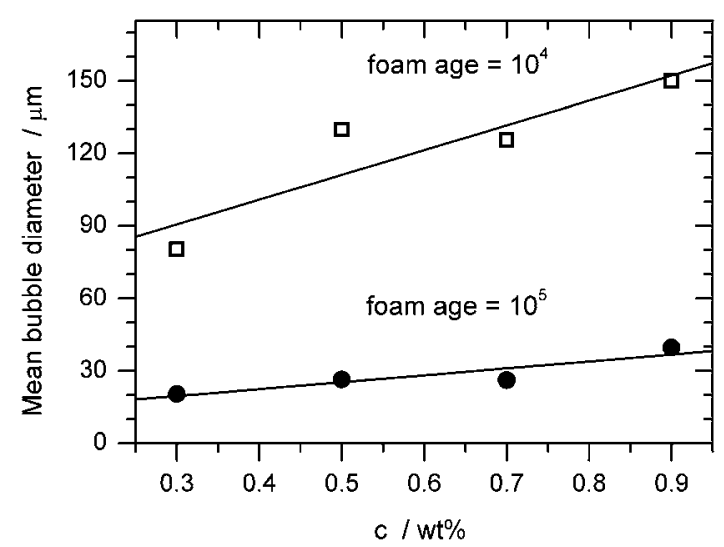

Fig. 4 Mean diameter obtained from the 3D bubble distributions of "wet" foams for different silica concentrations $(c=0.3,0.5,0.7$, and $0.9 \mathrm{wt} \%$ ) at foam ages $\approx 10^{4} \mathrm{~s}$ (squares) and $10^{5} \mathrm{~s}$ (circles). Lines are linear fits to the data.

Fig. 5 depicts a series of 3D tomograms and corresponding 2D slices of "wet" foams at a foam age of $\sim 10^{4} \mathrm{~s}$ with different silica concentrations $(c=0.3,0.5,0.7$, and $0.9 \mathrm{wt} \%$ ) obtained by shaking. The reduction of the number of bigger bubbles with increasing fraction of silica particles is qualitatively clearly visible from the tomographic images. The corresponding numberaveraged and volume-averaged bubble size distributions are shown in Fig. 6. The volume-averaged distributions (Fig. 6, inlays) give as expected mean diameters larger than the number-averaged distribution, and allow to better reveal the presence of a second population of large bubbles. Note that there are not many large bubbles in the images; hence the quality of the statistical averages in this range of diameters is poor. For each bulk concentration studied, Fig. 4 shows a significant decrease of the mean diameter of the small bubbles (by a factor of 3.8-4.9) between foam ages $=10^{4}$ and $10^{5} \mathrm{~s}$. Moreover, within the same foam age, we observed an increase of both homogeneity and mean bubble diameter when increasing the bulk concentration (Fig. 4 and 6). These surprising observations will be discussed later.

We also performed a few trials with dry foams. For $c<$ $0.7 \mathrm{wt} \%$, the foam evolution (Fig. 1) is too rapid to be followed by CT (measurement time $=c a$. 1 hour) and no reconstructions could be obtained. A lab CT $3 \mathrm{D}$ reconstruction for $c=0.7 \mathrm{wt} \%$, $\varepsilon \approx 0.09$ and foam age $\approx 10^{6} \mathrm{~s}$ is shown in Fig. 7(a). In this case the mean bubble diameter is $25 \mu \mathrm{m}$ (Fig. 7(b)). The large empty regions observable in the image are not caused by foam collapse or coarsening, but by incomplete filling due to some air trapped in the syringe during the preparation protocol. This artefact was taken into account for the quantitative analysis of the bubble size distribution. A magnification of the same foam, but dried $(\varepsilon \approx 0)$, by synchrotron CT is also shown in Fig. 7(c). In the latter magnification, the foam is directly in contact to the atmosphere and all the water is evaporated. The resulting foam structure is very fragile and it can be regarded as the solid particle template of the foam.
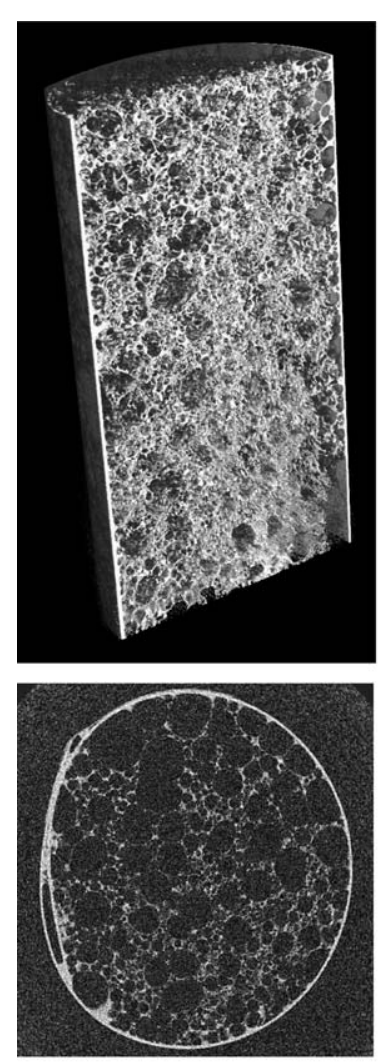

$0.3 \mathrm{wt} \%$

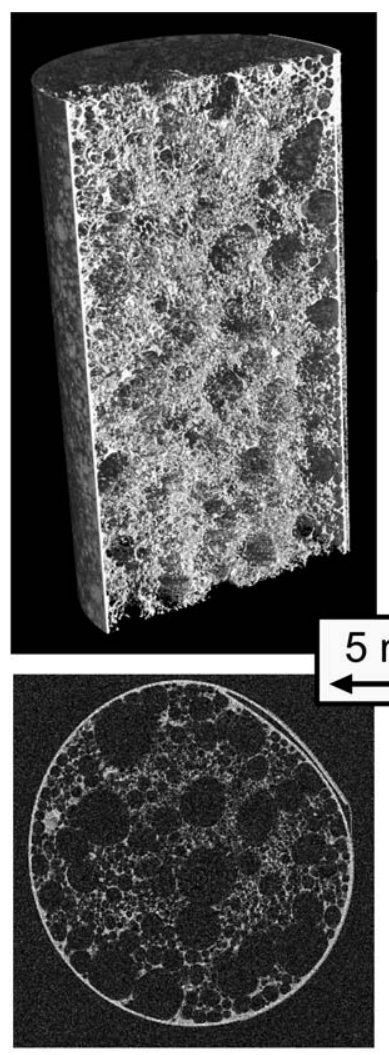

$0.5 w t \%$

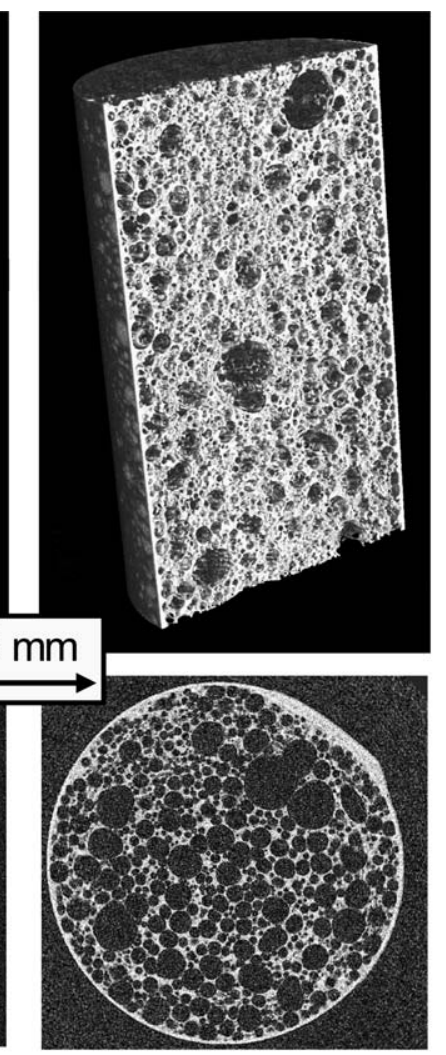

$0.7 w t \%$
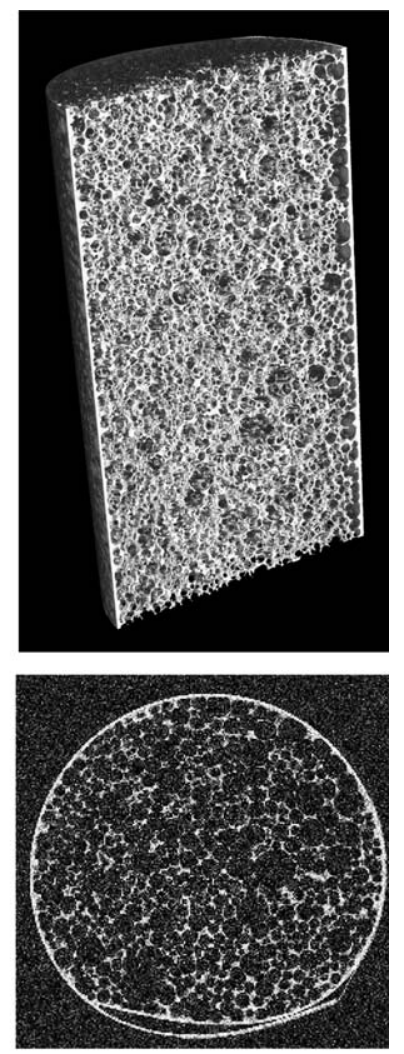

0.9 wt $\%$

Fig. 5 3D cut of X-ray $\mu$-CT image reconstructions (up) and 2D slice (down) of "wet" foams obtained by shaking for different bulk concentrations $(c=0.3,0.5,0.7$, and $0.9 \mathrm{wt} \%)$ at a foam age $\approx 10^{4} \mathrm{~s}$. 

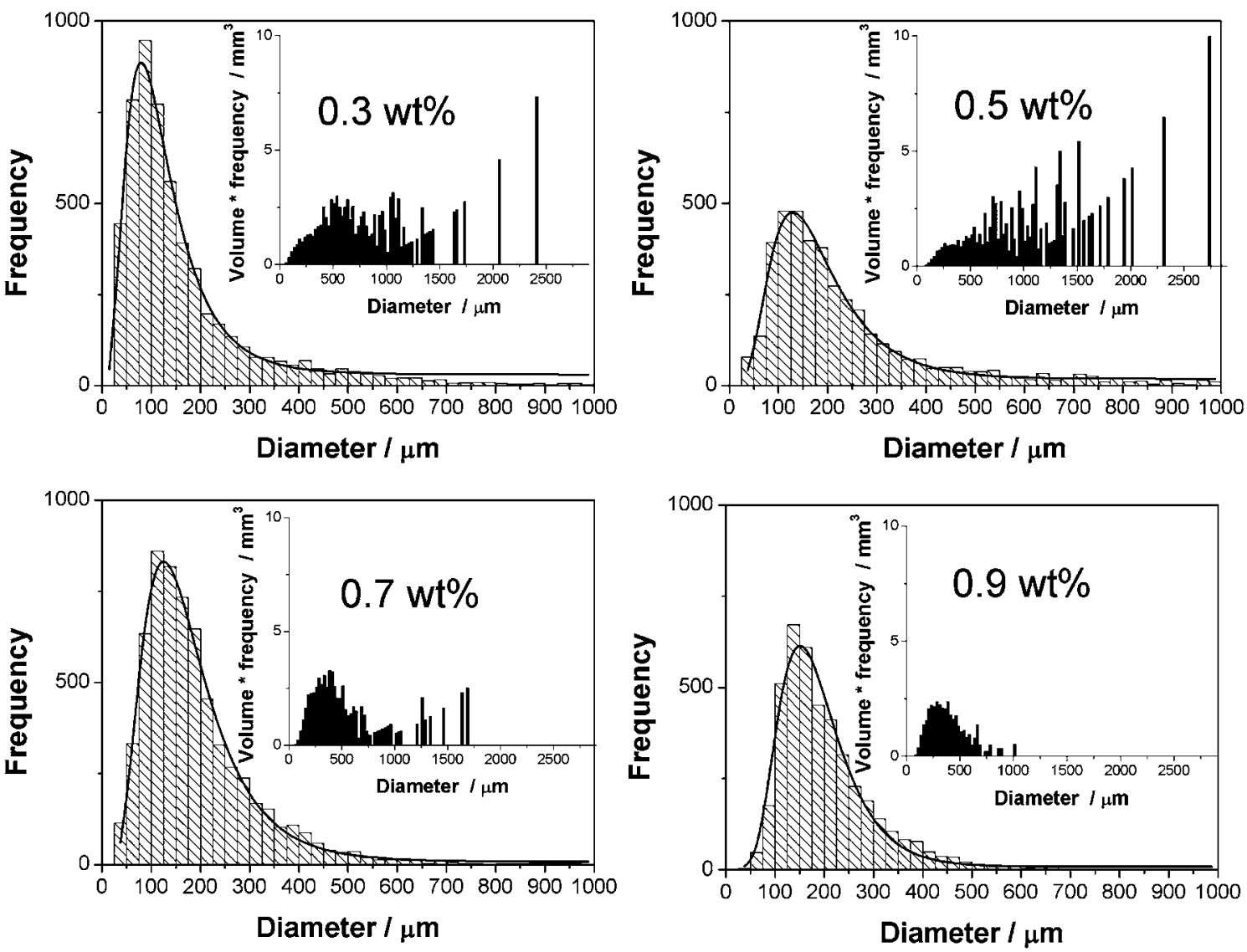

Fig. 6 Number- and volume-averaged (inlay) bubble size distributions of "wet" foams for different bulk concentrations $(c=0.3,0.5,0.7$, and 0.9 wt $\%)$ at a foam age $\approx 10^{4} \mathrm{~s}$.
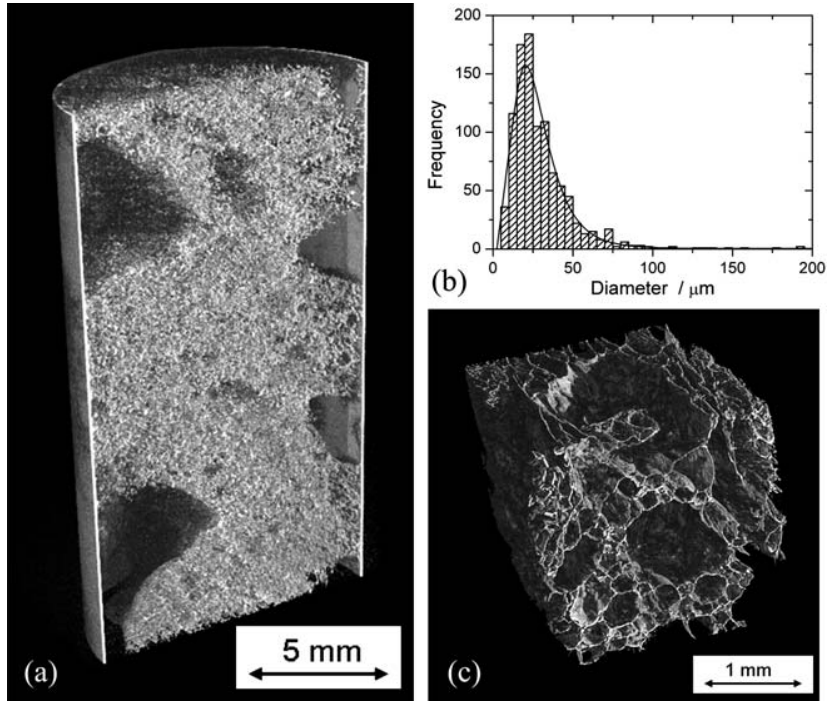

(b)

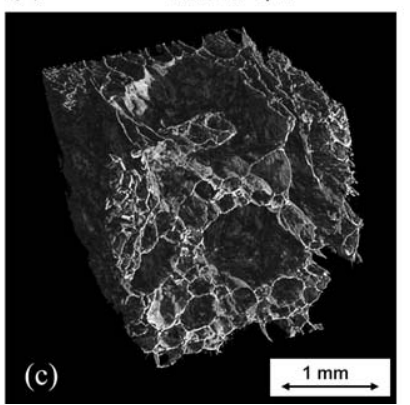

Fig. 7 (a) Lab 3D X-ray $\mu$-CT image reconstructions of a "dry" foam for $c=0.7 \mathrm{wt} \%$ at a foam age $\approx 10^{6} \mathrm{~s}$. (b) Number-averaged bubble size distribution. (c) High resolution $\mu$-CT synchrotron image.

\section{Discussion}

Foams evolve with time due to different mechanisms: drainage, coarsening (shrinking of small bubbles and growth of large bubbles due to gas transfer through the liquid driven by the difference of Laplace pressure between bubbles) and coalescence (rupture of liquid films between bubbles). ${ }^{1}$ The foams studied here drain as surfactant foams, but are much more stable, both against coarsening and coalescence.

The stability of the dry foams increases while increasing the bulk concentration of the dispersion (Fig. 1). $I_{\mathrm{S}}$ curves shift systematically towards longer times for $c=0.1,0.3,0.5 \mathrm{wt} \%$. For $c=0.7 \mathrm{wt} \%$, the limiting value of the intensity $\left(I_{\mathrm{s}} / I_{\mathrm{s}}{ }^{0}=c a .10\right)$ was not reached even after 8 days and the foam is virtually indefinitely stable. A mean bubble diameter of $25 \mu \mathrm{m}$ and a homogeneous size distribution were found at a foam age $\approx 10^{6} \mathrm{~s}$ (Fig. 7).

The threshold concentration of about $0.7 \mathrm{wt} \%$ above which coarsening stops is in perfect agreement with a recent study on particles laden interfaces: ${ }^{10}$ when $c$ increases, the surface tension $\gamma$ decreases and the interfacial compression elastic modulus $E$ increases. ${ }^{10}$ When $c \geq 0.7 \mathrm{wt} \%$, the Gibbs stability criterion $E>$ $\gamma / 2$ is fulfilled, expressing the fact that isolated bubbles are stable against coarsening. ${ }^{9,10}$ Above this threshold concentration, a network-like structure of nanoparticles forms at the air-water interface (as observed by Brewster angle microscopy) which may account for the large elastic modulus. ${ }^{10}$

Significantly different results were found for "wet" foams (Fig. 1 and 2). In this case $I_{\mathrm{S}}$ does not change in time and even tends to decrease in the first stage of aging (Fig. 2), pointing to a decrease of the mean bubble diameter. X-Ray computed 
tomography results show indeed a significant decrease of the mean bubble diameter during aging for all dispersion concentrations (Fig. 4). The final mean bubble diameter $=c a .25 \mu \mathrm{m}$, close to that found in dry foams. Furthermore, in Fig. 3, we can clearly see that aging causes also the formation of few larger bubbles.

The presence of these large bubbles invalidates the assumption made to analyze the multiple light scattering experiments, namely of homogeneous media in which the photon transport in the bulk can be described by a unique mean free path of light $l^{*}$. Following the light transport theory in opaque media, ${ }^{2}$ the effect of a bimodal distribution of bubble sizes on $I_{\mathrm{s}}$ can be estimated as follows. The effective mean free path is $l \approx 1 /(\rho \sigma)$, where $\rho$ is the density of scatterers and $\sigma$ is their cross-section, and in the case of two population $\left(s\right.$ and $b$ ) of bubble sizes $1 / l=\rho_{\mathrm{s}} \sigma_{\mathrm{s}}+\rho_{\mathrm{b}} \sigma_{\mathrm{b}}=1 / l_{\mathrm{s}}+$ $1 / l_{\mathrm{b}}{ }^{16}$ In foams, the main source of scattering is the plateau borders and $\sigma \approx r D$, where $r$ is the radius of curvature and $D$ is the length of the plateau border ( $\sim$ mean bubble diameter). Assuming that $r$ is the same in all cases (the large bubbles are surrounded by many small ones, see Fig. 3 and 5): $1 / l \approx \rho D$ and $1 / I_{\mathrm{s}} \approx \rho_{\mathrm{s}} D_{\mathrm{s}}+\rho_{\mathrm{b}} D_{\mathrm{b}}$. Let us discuss the case of $c=0.9 \mathrm{wt} \%$ : at $\sim 10^{4} \mathrm{~s}$ there is one main population of bubbles with $D_{0}=150 \mu \mathrm{m}$ whereas at $\sim 10^{5} \mathrm{~s}$, there are two populations, small bubbles with $D_{\mathrm{s}}=40 \mu \mathrm{m}$ and large bubbles with $D_{\mathrm{b}} \approx 1 \mathrm{~mm}$ (Fig. 3 and 4 ). Since $I_{\mathrm{s}}$ remains approximately constant, one has: $D_{0}=$ $D_{\mathrm{s}}(1-\varphi)+D_{\mathrm{b}} \varphi$, with $\varphi=\rho_{\mathrm{b}} /\left(\rho_{\mathrm{s}}+\rho_{\mathrm{b}}\right)$, leading to $\varphi \approx 10 \%$ in reasonable agreement with the $\mathrm{X}$-ray tomographic images (Fig. 2). Thus, the expected decrease for $I_{\mathrm{s}}$ due to the decreasing small bubbles diameter (Fig. 3) is likely balanced by the presence of a few large bubbles ( $\sim 1 \mathrm{~mm}$ diameter, Fig. 3$)$

As can be seen in Fig. 3-6, the increase of number of big bubbles is correlated with a reduction of the mean diameter of the small bubbles. The presence of the big bubbles is better seen in the volume-averaged distribution (Fig. 6), which also shows that there are fewer big bubbles at larger bulk concentration. These observations suggests that we are observing the first steps of a coarsening process, in which the small bubbles shrink and tend to disappear, while the larger bubbles grow. Here the small bubbles do not completely disappear; on the contrary, having reached a lower critical diameter of about $25 \mu \mathrm{m}$ (Fig. 4), they remain stable. The scenario depicted before resembles the mechanism suggested by Ashby and Binks for Laponite stabilised Pickering emulsions. The interfacial tension for particle stabilized bubble, in fact, decreases when the size of the bubble decreases and the Laplace pressure for small bubbles can be the same as for big bubbles. ${ }^{17}$

The fact that the bubble size is larger at larger concentration is very counter-intuitive, but can be rationalised in the same way: the elastic modulus $E$ of particle layers formed with dilute dispersions is small, hence coarsening is fast, and the diameters found for the population of small bubbles are smaller than those obtained with more concentrated dispersions, for which $E$ is larger. It was indeed shown that the rate of coarsening decreases when $E$ increases. ${ }^{18,19}$

Note that the big bubbles can also originate from film rupture (coalescence). Indeed, in the course of foam ageing small bubbles remain more stable than bigger ones: the probability of coalescence is proportional to the surface area and is larger for larger bubbles. Furthermore, particle layers rupture under expansion, whereas they buckle upon compression: ${ }^{20}$ the cohesion of the surface layers covering the large bubbles can therefore be lost and coalescence facilitated. The pictures show that there is a small number of extremely large bubbles after long aging times, that could arise from both coarsening and coalescence.

Let us finally compare the results obtained for "dry" and "wet" foams at long times. For aging time $\geq 10^{5} \mathrm{~s}$ and $c=0.7 \mathrm{wt} \%$, the average bubble size for the "dry" foam, $D=25 \mu \mathrm{m}$, is very close to $D=26 \mu \mathrm{m}$ for the "wet" foam. Although bubble sizes are close, foam initial liquid fractions are quite different: $\varepsilon_{0} \approx 0.34$ and $\varepsilon_{0} \approx 0.18$ respectively. The concentration at which foams become very stable is also different: $c>0.3 \mathrm{wt} \%$ for foams produced by simple shaking $\left(\varepsilon_{0} \approx 0.34\right)$ and $c>0.7 \mathrm{wt} \%$ for foams produced by turbulent mixing $\left(\varepsilon_{0} \approx 0.18\right)$ corresponding in both cases to a particle concentration in the foam $c_{\mathrm{f}}=\varepsilon \times c \approx$ $0.1 \mathrm{wt} \%$ (in the approximation of a negligible amount of particle in the drained liquid).

The fact that the final bubble size is independent of the procedure used to make the foam suggests that this size is controlled by an optimal surface coverage provided by the particles. We can estimate this coverage by considering that the bubbles have the shape of tetrakaidecahedron (Kelvin cell) and use the known calculations of cell volume. ${ }^{21}$ The surface concentration is then $\Gamma_{\mathrm{f}}=c_{\mathrm{f}} \times l_{\mathrm{v}} \times 11.31 / 26.8$, where $l_{\mathrm{v}}$ is the length of the vertices of hexagonal faces $=0.36 \mathrm{D}$. It follows that $\Gamma_{\mathrm{f}} \approx 4 \mathrm{mg} \mathrm{m}^{-2}$. The latter concentration is not very different when one assumes spherical bubble geometry: $\Gamma_{\mathrm{f}}=c_{\mathrm{f}} \times D / 6$. Note that $\Gamma_{\mathrm{f}}$ is significantly lower than a fully packed concentration estimated from the primary particle size $\left(\sim 50 \mathrm{mg} \mathrm{m}^{-2}\right)$. Hence, $\Gamma_{\mathrm{f}}$ can be regarded as a percolation concentration for the formation of a loose interfacial network of particles able to arrest coarsening. ${ }^{22}$

\section{Conclusions}

We have shown that aqueous foams stabilised by nanoparticles can be easily imaged using an X-ray laboratory source. We have used hydrophobically modified silica nanoparticles that confer to the foam a remarkable stability. The X-ray tomography observations were compared with the information obtained using a multiple light scattering technique.

Both techniques confirm that provided the concentration of particles in foams is high enough, the bubble size evolves little with time. For relative wet foams, X-ray tomography revealed the presence of two populations of bubbles, a large number of small bubbles which size tends to decrease with time and a small number of large bubbles which number tends to increase with time. These observations suggests that we are observing the first steps of a coarsening process, in which the small bubbles shrink and tend to disappear, while the larger bubble grow. In the case of foams made with concentrated particle dispersions the process seems to halt when the diameter of the small bubbles reaches values around $30 \mu \mathrm{m}$. More work remains to be done to understand better this particular coarsening process. These results demonstrate the potential of the two techniques and of their combination, and open interesting perspectives for further foam studies. 


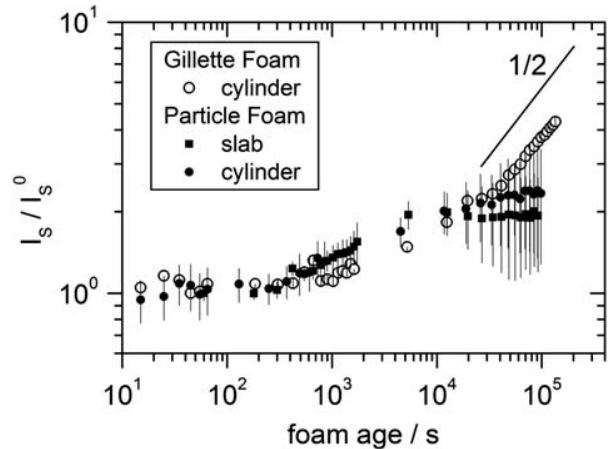

Fig. 8 Multiple scattered intensity ratio as a function of time for a Gillette foam and a "dry" foam $(c=0.7 \mathrm{wt} \%)$ in a slab-shaped (squares) and a cylindrical container (circles).

\section{Appendix}

\section{Experimental validation of the multiple light scattering analysis}

In order to test the multiple light scattering setup and the effect of the sample container geometry, we measured $I_{\mathrm{s}}$ for a foam with known properties (Gillette Normal, UK; $\varepsilon_{0} \approx 0.08$, initial mean bubble diameter $\approx 30 \mu \mathrm{m})$ in a cylindrical container $(L=$ $27 \mathrm{~mm}$ ), and $I_{\mathrm{s}}$ for a particle stabilised foam obtained by turbulent mixing at $c=0.7 \mathrm{wt}^{\mathrm{t}} \%$ both in a slab-shaped ( $L=$ $25 \mathrm{~mm})$ and in a cylindrical container $(L=27 \mathrm{~mm})$. The results are summarized in Fig. 8 where the ratio between the multiply scattered intensity $I_{\mathrm{s}}$ and its initial value $I_{\mathrm{s}}{ }^{0}$ (intensity extrapolated to zero foam age) is plotted as a function of time. For the Gillette normal foam, $I_{\mathrm{s}}$ increases following the asymptotic theoretical scaling of $1 / 2$ is in good agreement with the data reported by Durian et al. for a slab-shaped sample container. ${ }^{4}$ For particle stabilised foams, $I_{\mathrm{s}}$ is essentially constant and the data for slab and curved geometries overlap within the experimental errors, thus, confirming the negligible effect of the container geometry on the scattering results as predicted theoretically by Vanel et $a .^{23}$

\section{Acknowledgements}

We thank Wacker-Chemie (Burghausen) for kindly providing the fumed silica powders. We also thank Andreas Benz for the support in the tomographic analysis and Arnaud Saint-Jalmes and Wiebke Drenckhan for fruitful discussions. ESA support (MAP: MicroG-foam, XRMON and Hydrodynamics of Wet Foams) is gratefully acknowledged.

\section{References}

1 D. Weaire and S. Hutzler, The Physics of Foams, OUP Oxford, UK, 2001.

2 M. Vera, A. Saint-Jalmes and D. Durian, Appl. Opt., 2001, 40, 42104214.

3 D. Pine, D. Weitz, P. Chaikin and E. Herbolzheimer, Phys. Rev. Lett., 1998, 60, 1134-1137.

4 D. Durian, D. Weitz and D. Pine, Phys. Rev. A: At., Mol., Opt. Phys,, 1991, 44, 7902-7905.

5 M. Vera and D. Durian, Phys. Rev. E: Stat. Phys., Plasmas, Fluids, Relat. Interdiscip. Top., 1996, 53, 3215-3224.

6 J. Banhart, Advanced Tomographic Methods in Materials Research and Engineering, Oxford University Press, 2008.

7 J. Lambert, I. Cantat, R. Delannay, R. Mokso, P. Cloetens, J. A. Glazier and F. Graner, Phys. Rev. Lett., 2007, 99, 058304.

8 J. Lambert, I. Cantat, R. Delannay, A. Renault, F. Graner, J. A. Glazier, I. Veretennikov and P. Cloetens, Colloids Surf., A, 2005, 263, 295-302.

9 A. Cervantes-Martinez, E. Rio, G. Delon, A. Saint-Jalmes, D. Langevin and B. P. Binks, Soft Matter, 2008, 4, $1531-1535$.

10 A. Stocco, W. Drenckhan, E. Rio, D. Langevin and B. P. Binks, Soft Matter, 2009, 5, 2215-2222.

11 A. Saint-Jalmes, M. Vera and D. Durian, Eur. Phys. J. B, 1999, 12, 67-73.

$12 \mathrm{http} / / / \mathrm{www} . x \mathrm{raylab} . \mathrm{com} /$.

$13 \mathrm{http}: / /$ www.volumegraphics.com/products/vgstudiomax/.

14 http://www.vsg3d.com/.

15 A. Saint-Jalmes, Soft Matter, 2006, 2, 836-849.

16 D. Pine, D. Weitz, J. Zhu and E. Herbolzheimer, J. Phys. (Paris), 1990, 51, 2101-2127.

17 N. Ashby and B. P. Binks, Phys. Chem. Chem. Phys., 2000, 2, $5640-5646$.

18 D. Georgieva, A. Cagna and D. Langevin, Soft Matter, 2009, 5, 2063-2071.

19 W. Kloek, T. van Vliet and M. Meinders, J. Colloid Interface Sci., 2001, 237, 158-166.

20 M. Safouane, D. Langevin and B. P. Binks, Langmuir, 2007, 23, $11546-11553$.

21 L. J. Gibson and M. F. Ashby, Cellular Solids, Cambridge Univ.Press, Cambridge, UK, 1997.

22 T. Horozov, Curr. Opin. Colloid Interface Sci., 2008, 13, 134-140.

23 L. Vanel, P. Lemieux and D. Durian, Appl. Opt., 2001, 40, $4179-4186$ 